Kragujevac Journal of Mathematics

Volume 45(5) (2021), PAges 721-738.

\title{
WARPED PRODUCT POINTWISE SEMI-SLANT SUBMANIFOLDS OF SASAKIAN MANIFOLDS
}

\author{
ION MIHAI ${ }^{1}$, SIRAJ UDDIN ${ }^{2}$, AND ADELA MIHA
}

Abstract. Recently, B.-Y. Chen and O. J. Garay studied pointwise slant submanifolds of almost Hermitian manifolds. By using the notion of pointwise slant submanifolds, we investigate the geometry of pointwise semi-stant submanifolds and their warped products in Sasakian manifolds. We givenon-trivial examples of such submanifolds and obtain several fundamental results, including a characterization for warped product pointwise semi-slant submanifolds of Sasakian manifolds.

\section{INTRODUC}

In [7], B.-Y. Chen introduced the notion of slant submanifolds of almost Hermitian manifolds as a natural generalization of holonorphic (invariant) and totally real (antiinvariant) submanifolds. Adterwards, the geometry of slant submanifolds became an active topic of research in differential geometry. Later, A. Lotta [20] has extended this study for almost contact metric manifolds. J. L. Cabrerizo et al. investigated slant submanifolds of a Sasakian manifold [6]. N. Papaghiuc introduced in [22] a class of submanifolds, called semi-slant submanifolds of almost Hermitian manifolds, which are the generalizations of slant and CR-submanifolds. Later on, Cabrerizo et al. [5] extended this idea for semi-slant submanifolds of contact metric manifolds and providee many exampres of such submanifolds.

Next, as an extension of slant submanifolds of an almost Hermitian manifold, F. Etayo [16] introdúced the notion of pointwise slant submanifolds of almost Hermitian manifolds. B.-Y. Chen and O. J. Garay [14] studied pointwise slant submanifolds of almost Hermitian manifolds. They have obtained several fundamental results, in

Key words and phrases. Warped products, pointwise slant submanifolds, pointwise semi-slant submanifolds, Sasakian manifolds.

2010 Mathematics Subject Classification. Primary: 53C15, 53C40, 53C42. Secondary: $53 \mathrm{~B} 25$.

DOI 10.46793/KgJMat2105.721M

Received: March 13, 2019.

Accepted: May 09, 2019. 
particular, a characterization of these submanifolds. K. S. Park [23] has extended this study. B. Sahin studied pointwise semi-slant submanifolds and warped product pointwise semi-slant submanifolds by using the notion of pointwise slant submanifolds [26]. In [31], the authors considered pointwise slant submanifolds of an almost contact metric manifold such that the structure vector field $\xi$ is tangent to the submanifold. They have obtained a simple characterization for such submanifolds and studied warped product pointwise pseudo-slant submanifolds of Sasakian manifolds.

In 1969, R. L. Bishop and B. O'Neill [3] introduced and studied warped product manifolds. 30 years later, around the beginning of this century, B.-Y. Chen initiated in $[9,10]$ the study of warped product CR-submanifolds of Kaehler manifolds. Chen's work in this line of research motivated many geometers to study the geometry of warped product submanifolds by using his idea for different structures on manifolds (see, for instance, $[2,17,21]$ and [27]). For a detailed survey on warped product submanifolds we refer to Chen's books $[11,13]$ and his suryey article $[12$ as well.

In [24], B. Sahin showed that there exists no proper warped product semi-slant submanifold of Kaehler manifolds. Then, he introduced the notion of warped product hemi-slant submanifolds of Kaehler manifolds [25]. He defined and studied warped product pointwise semi-slant submanifolds and showed that there exists a non-trivial warped product pointwise semi-slant submanifold of the form $M_{T} \times_{f} M_{\theta}$ in a Kaehler manifold $\tilde{M}$, where $M_{T}$ and $M_{\theta}$ are invariant and proper pointwise slant submanifolds of $\tilde{M}$, respectively [26]. For almost contact metric manifolds, we have seen in [19] and [1] that there are no proper warped product semi-slant submanifolds in cosymplectic and Sasakian manifolds. Then, we have considered warped product pseudo-slant submanifolds (warped product hemi-slant submanifolds [25], in the same sense of almost Hermitian manifolds) of cosymplectic [28] and Sasakian manifolds [29].

K. S. Park [23] studied warped product pointwise semi-slant submanifolds. He proved that there do not exist warped product pointwise semi-slant submanifolds of the form $M_{\theta} \times_{f} M_{T}$ such that $M_{\theta}$ and $M_{T}$ are proper pointwise slant and invariant submanifolds, respectively. Then he provided many examples and obtained several results for warped products by reversing these two factors, including sharp estimations for the squared norm of the second fundamental form in terms of the warping functions. Later, we also extended this idea in [31] to warped product pointwise pseudo-slant submanifolds of Sasakian manifolds.

In this paper, study warped product pointwise semi-slant submanifolds of the form $M_{T} \times M_{\theta}$ of Sasakian manifolds.

The present paper is organized as follows: in Section 2, we give basic definitions and formulas needed for this paper. Section 3 is devoted to the study of pointwise semislant submanifolds of Sasakian manifolds; we define pointwise semi-slant submanifolds and in the definition of pointwise semi-slant submanifolds we assume that the structure vector field $\xi$ is always tangent to the submanifold. We give two non-trivial examples of such submanifolds for the justification of our definition and a result which is useful to the next section. In Section 4, we study warped product pointwise semi-slant 
submanifolds of Sasakian manifolds. In [1], we have seen that there are no warped product semi-slant submanifolds of the form $M_{T} \times{ }_{f} M_{\theta}$ in a Sasakian manifold other than contact CR-warped products, but if we assume that $M_{\theta}$ is a proper pointwise slant submanifold then there exists a non-trivial class of such warped products. In this section, we obtain several new results which are generalizations of warped product semi-slant submanifolds and contact CR-warped product submanifolds. In Section 5, we provide nontrivial examples of Riemannian product and warped product pointwise semi-slant submanifolds in Euclidean spaces.

\section{Preliminaries}

An almost contact structure $(\varphi, \xi, \eta)$ on a $(2 n+1)$-dimensional manifold $\tilde{M}$ is defined by a $(1,1)$ tensor field $\varphi$, a vector field $\xi$, called characteristic or Reeb vector field, and a 1-form $\eta$ satisfying the following conditions

$$
\varphi^{2}=-I+\eta \otimes \xi, \quad \eta(\xi)=1, \quad \eta \circ \xi=0, \quad(\xi)
$$

where $I: T \tilde{M} \rightarrow T \tilde{M}$ is the identity map [4]. There always exists a Riemannian metric $g$ on an almost contact manifold $\tilde{M}$ satisfying the following compatibility condition

$$
g(\varphi X, \varphi Y)=g(X, Y)-\eta(X) \eta(Y)
$$

for any $X, Y \in \Gamma(T \tilde{M})$, the Lie algebra of vector fields on $\tilde{M}$. This metric $g$ is called a compatible metric and the manifold $\tilde{M}$ together with the structure $(\varphi, \xi, \eta, g)$ is called an almost contact metric manifold. As an immediate consequence of (2.2), one has $\eta(X)=g(X, \xi)$ and $g(\varphi X, Y)=g(X, \varphi Y)$. If $\xi$ is a Killing vector field with respect to $g$, then the contact metric structure is called a $K$-contact structure. A normal contact metric manifold is said to be a Sasakian manifold. In terms of the covariant derivative of $\varphi$, the Sasakian condition can be expressed by

$$
\left(\tilde{\nabla}_{X} \varphi\right) \hat{Y}=g(X, Y) \xi-\eta(Y) X
$$

for all $X, Y \in \Gamma(T M)$ where $\tilde{\nabla}$ is the Levi-Civita connection of $g$. From the formula (2.3), it follows that

$$
\tilde{\nabla}_{X} \xi=-\varphi X
$$

for any $X \in \Gamma(T \tilde{y})$.

Let $M$ be a Riemannian manifold isometrically immersed in $\tilde{M}$ and denote by the same symbol $g$ the Riemannian metric induced on $M$. Let $\Gamma(T M)$ be the Lie algebra of vector fields in $M$ and $\Gamma\left(T^{\perp} M\right)$ the set of all vector fields normal to $M$. The Gauss and Weingarten formulas are respectively given by

$$
\tilde{\nabla}_{X} Y=\nabla_{X} Y+h(X, Y)
$$

and

$$
\tilde{\nabla}_{X} N=-A_{N} X+\nabla_{X}^{\perp} N
$$


for any $X, Y \in \Gamma(T M)$ and $N \in \Gamma\left(T^{\perp} M\right)$, where $\nabla$ is the Levi-Civita connection on $M, \nabla^{\perp}$ is the normal connection in the normal bundle $T^{\perp} M$ and $A_{N}$ is the shape operator of $M$ with respect to the normal vector $N$. Moreover, $h: T M \times T M \rightarrow T^{\perp} M$ is the second fundamental form of $M$ in $\tilde{M}$. Furthermore, $A_{N}$ and $h$ are related by $[32]$

$$
g(h(X, Y), N)=g\left(A_{N} X, Y\right),
$$

for any $X, Y \in \Gamma(T M)$ and $N \in \Gamma\left(T^{\perp} M\right)$.

For any $X$ tangent to $M$, we write

$$
\varphi X=P X+F X
$$

where $P X$ and $F X$ are the tangential and normal components of $\varphi X$, respectively. Then $P$ is an endomorphism of the tangent bundle $T M$ and $F$ is a normal bundle valued 1-form on $T M$. Similarly, for any vector field $N$ nornal to $M$, we put

$$
\varphi N=t N+f N
$$

where $t N$ and $f N$ are the tangential and normal components of $\varphi N$, respectively. Moreover, from (2.2) and (2.8), we have

for any $X, Y \in \Gamma(T M)$.

Throughout this paper, we assume the structure field $\xi$ is tangent to $M$; otherwise $M$ is a C-totally real submanifold [20]. Let Me a Riemannian manifold isometrically immersed in an almost contact metric manifold $(\tilde{M}, \varphi, \xi, \eta, g)$. A submanifold $M$ of an almost contact metric manifold $\tilde{M}$ is said to be slant [6], if for each non-zero vector $X$ tangent to $M$ at $p \in M$ such that $X$ is not proportional to $\xi_{p}$, the angle $\theta(X)$ between $\varphi X$ and $T_{p} M$ is constant, i.e. it does not depend on the choice of $p \in M$ and $X \in T_{p} M-\left\langle\xi_{p}\right\rangle$

A slant submanifold is said to be proper slant if neither $\theta=0$ nor $\theta=\frac{\pi}{2}$. We note that on a slant submanifold if $\theta=0$, then it is an invariant submanifold and if $\theta=\frac{\pi}{2}$, then it is an anti-invariant submanifold. A slant submanifold is said to be proper slant if it is neither invariant nor anti-invariant.

As a natural extension of slant submanifolds, F. Etayo [16] introduced pointwise slant submanifolds of an almost Hermitian manifold under the name of quasi-slant submanifolds. Later on, B.-Y. Chen and O. J. Garay studied pointwise slant submanifolds of almost Hermitian manifolds and obtained many interesting results [14]. In [31], the authors studied pointwise slant submanifolds of almost contact metric manifolds tangent to the structure vector field $\xi$.

A submanifold $M$ of an almost contact metric manifold $\tilde{M}$ is said to be pointwise slant if for any nonzero vector $X$ tangent to $M$ at $p \in M$, such that $X$ is not propotional to $\xi_{p}$, the angle $\theta(X)$ between $\varphi X$ and $T_{p}^{*} M=T_{p} M-\{0\}$ is independent of the choice of nonzero vector $X \in T_{p}^{*} M$. In this case, $\theta$ can be regarded as a function on $M$, which is called the slant function of the pointwise slant submanifold. 
We note that every slant submanifold is a pointwise slant submanifold, but the converse is not true. We also note that a pointwise slant submanifold is invariant (respectively, anti-invariant) if for each point $p \in M$, the slant function $\theta=0$ (respectively, $\theta=\frac{\pi}{2}$ ). A pointwise slant submanifold is slant if and only if the slant function $\theta$ is constant on $M$. Moreover, a pointwise slant submanifold is proper if neither $\theta=0, \frac{\pi}{2}$ nor $\theta$ is constant.

In [31], we have obtained the following characterization theorem.

Theorem 2.1 ([31]). Let $M$ be a submanifold of an almost contact metric manifold $\tilde{M}$ such that $\xi \in \Gamma(T M)$. Then, $M$ is pointwise slant if and only if

$$
P^{2}=\cos ^{2} \theta(-I+\eta \otimes \xi),
$$

for some real valued function $\theta$ defined on the tangent bundle

The following relations are immediate consequences of Theorem 2.1 .

Let $M$ be a pointwise slant submanifold of an almost eontact metric manifold $\tilde{M}$. Then, we have

for any $X, Y \in \Gamma(T M)$.

The next useful relations for a pointwise stant submanifold of an almost contact metric manifold was obtained in [31]

for any $X \in \Gamma(T M)$.

$$
\begin{aligned}
& g(P X, P Y)=\cos ^{2} \theta[g(X, Y)-\eta(X) \eta(Y)], \\
& g(F X, F Y)=\sin ^{2} \theta[g(X, Y)-\eta(X),(Y)],
\end{aligned}
$$

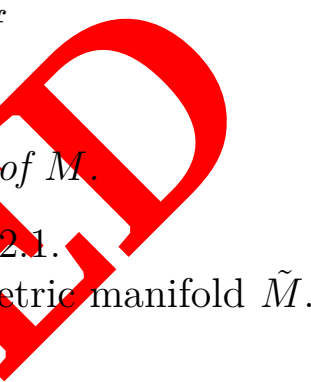

B. Sahin [26] defined and studred pointwise semi-slant submanifolds of Kaehler manifolds. In this section, we define and study pointwise semi-slant submanifolds of Sasakian manifolds

Definition 3.1. A submanifold $M$ of an almost contact metric manifold $\tilde{M}$ is said to be a pointwise semi-slant submanifold if there exists a pair of orthogonal distributions $\mathfrak{D}$ and $\mathfrak{D}^{\theta}$ on $M$ such that

(i) the tangent bundle $T M$ admits the orthogonal direct decomposition $T M=$ $\mathfrak{D} \oplus \mathfrak{D}^{\theta} \oplus\langle\xi\rangle ;$

(ii) the distribution $\mathfrak{D}$ is invariant under $\varphi$, i.e., $\varphi(\mathfrak{D})=\mathfrak{D}$;

(iii) the distribution $\mathfrak{D}^{\theta}$ is pointwise slant with slant function $\theta$.

Note that the normal bundle $T^{\perp} M$ of a pointwise semi-slant submanifold $M$ is decomposed as

$$
T^{\perp} M=F \mathfrak{D}^{\theta} \oplus \nu, \quad F \mathfrak{D}^{\theta} \perp \nu,
$$


where $\nu$ is an invariant normal subbundle of $T^{\perp} M$ under $\varphi$.

If we denote the dimensions of $\mathfrak{D}$ and $\mathfrak{D}^{\theta}$ by $m_{1}$ and $m_{2}$, respectively, then we have the following.

(i) If $m_{1}=0$, then $M$ is a pointwise slant submanifold.

(ii) If $m_{2}=0$, then $M$ is an invariant submanifold.

(iii) If $m_{1}=0$ and $\theta=\frac{\pi}{2}$, then $M$ is an anti-invariant submanifold.

(iv) If $m_{1} \neq 0$ and $\theta=\frac{\pi}{2}$, then $M$ is a contact CR-submanifold.

(v) If $\theta$ is constant on $M$, then $M$ is a semi-slant submanifold with slant angle $\theta$.

We also note that a pointwise semi-slant submanifold is proper if neither $m_{1}, m_{2}=0$ nor $\theta=0, \frac{\pi}{2}$ and $\theta$ should not be a constant.

Now, we provide the following non-trivial examples of pointwise sem-slant submanifolds of an almost contact metric manifold.

Example 3.1. Let $\left(\mathbf{R}^{7}, \varphi, \xi, \eta, g\right)$ be an almost contact metriemanifold with cartesian coordinates $\left(x_{1}, y_{1}, x_{2}, y_{2}, x_{3}, y_{3}, z\right)$ and the almost

$$
\varphi\left(\frac{\partial}{\partial x_{i}}\right)=-\frac{\partial}{\partial y_{i}}, \quad \varphi\left(\frac{\partial}{\partial y_{j}}\right)=\frac{\partial}{\partial x_{j}}, \quad \varphi\left(\frac{\partial}{\partial z}\right)=0, \quad 1 \leq i, j \leq 3,
$$

where $\xi=\frac{\partial}{\partial z}, \eta=d z$ and $g$ is the standard Euclidean metric on $\mathbf{R}^{7}$. Then $(\varphi, \xi, \eta, g)$ is an almost contact metric structure on $\mathbf{R}^{7}$. Consider a submanifold $M$ of $\mathbf{R}^{7}$ defined by $\psi(u, v, w, t, z)=(u+v,-u+v, t \cos w, t \sin w, w \cos t, w \sin t, z)$, such that $w, t(w \neq t)$ are non-zero realnumbers. Then the tangent space TM is spanned by the following vector fields

$$
\begin{aligned}
& X_{1}=\frac{\partial}{\partial x_{1}}-\frac{\partial}{\partial y_{1}} X_{2}=\frac{\partial}{\partial x_{1}}+\frac{\partial}{\partial y_{1}}, \\
& X_{3}=-t \sin w \frac{\partial}{\partial x_{2}}+t \cos w \frac{\partial}{\partial y_{2}}+\cos t \frac{\partial}{\partial x_{3}}+\sin t \frac{\partial}{\partial y_{3}}, \\
& X_{4}=\cos w \frac{\partial}{\partial x_{2}} \sin w \frac{\partial}{\partial y_{2}}-w \sin t \frac{\partial}{\partial x_{3}}+w \cos t \frac{\partial}{\partial y_{3}}, \quad X_{5}=\frac{\partial}{\partial z} .
\end{aligned}
$$

Thus, we observe that $\mathfrak{D}=\operatorname{Span}\left\{X_{1}, X_{2}\right\}$ is an invariant distribution and $\mathfrak{D}^{\theta}=$ $\operatorname{Span}\left\{X_{3}, X_{4}\right\}$ is a pointwise slant distribution with pointwise slant function $\theta=$ $\cos ^{-1}\left((t-w) / \sqrt{\left(t^{2}+1\right)\left(w^{2}+1\right)}\right)$. Hence, $M$ is a pointwise semi-slant submanifold of $\mathbf{R}^{7}$ such that $\xi=\frac{\partial}{\partial z}$ is tangent to $M$.

Example 3.2. Consider a submanifold of $\mathbf{R}^{7}$ with almost contact structure $\varphi$ given in Example 3.1. If the immersion $\psi: \mathbf{R}^{5} \rightarrow \mathbf{R}^{7}$ is given by

$$
\psi\left(u_{1}, u_{2}, u_{3}, u_{4}, t\right)=\left(u_{1}, \frac{u_{3}^{2}+u_{4}^{2}}{2}, \cos u_{4},-u_{2}, \frac{u_{3}^{2}-u_{4}^{2}}{2}, \sin u_{4}, t\right), \quad u_{4} \neq 0,
$$


then the tangent space $T M$ is spanned by $X_{1}, X_{2}, X_{3}, X_{4}$ and $X_{5}$, where

$$
\begin{aligned}
& X_{1}=\frac{\partial}{\partial x_{1}}, \quad X_{2}=-\frac{\partial}{\partial y_{1}}, \quad X_{3}=u_{3} \frac{\partial}{\partial x_{2}}+u_{3} \frac{\partial}{\partial y_{2}}, \\
& X_{4}=u_{4} \frac{\partial}{\partial x_{2}}-u_{4} \frac{\partial}{\partial y_{2}}-\sin u_{4} \frac{\partial}{\partial x_{3}}+\cos u_{4} \frac{\partial}{\partial y_{3}}, \quad X_{5}=\frac{\partial}{\partial t} .
\end{aligned}
$$

Therefore, $M$ is a pointwise semi-slant submanifold such that $\mathfrak{D}=\operatorname{Span}\left\{X_{1}, X_{2}\right\}$ is an invariant distribution and $\mathfrak{D}^{\theta}=\operatorname{Span}\left\{X_{3}, X_{4}\right\}$ is a pointwise slant distribution with pointwise slant function $\theta=\cos ^{-1}\left(\sqrt{2} u_{4} / \sqrt{1+2 u_{4}^{2}}\right)$.

Now, we obtain the following useful results for semi-slant submanifolds of a Sasakian manifold.

Lemma 3.1. Let $M$ be a pointwise semi-slant submanifold of a Sasakian manifold $\tilde{M}$. Then, we have

(i) $\sin ^{2} \theta g\left(\nabla_{X} Y, Z\right)=g(h(X, \varphi Y), F Z)-g(h(X, Y), F P Z)$,

(ii) $\sin ^{2} \theta g\left(\nabla_{Z} W, X\right)=g(h(X, Z), F P W)-g(h(\boldsymbol{X}, \mathbf{Z}), F W)$,

for any $X, Y \in \Gamma(\mathfrak{D} \oplus\langle\xi\rangle)$ and $Z, W \in \Gamma\left(\mathfrak{D}^{\theta}\right)$.

Proof. The first and second parts of the lemma can be proved in a similar way. For any $X, Y \in \Gamma(\mathfrak{D} \oplus\langle\xi\rangle)$ and $Z \in \Gamma\left(\mathfrak{D}^{\theta}\right)$, we have

$$
g\left(\nabla_{X} Y, Z\right)=g\left(\tilde{\nabla}_{X} Y\right)=g\left(\varphi \tilde{\nabla}_{X} Y, \varphi Z\right) .
$$

From the covariant derivative formula of $\varphi$, we derive

$$
g\left(\nabla_{X} Y, Z\right)=g\left(\tilde{\nabla}_{X} \varphi Y, \varphi Z\right)-g\left(\left(\tilde{\nabla}_{X} \varphi\right) Y, \varphi Z\right) .
$$

Then, from (2.3), (2.8) and the orthogonality of the two distributions, we find

Again, from the covariant derivative formula of $\varphi$, we get

$$
\begin{aligned}
g\left(\nabla_{X} Y, Z\right) & =g\left(\tilde{\nabla}_{X} \varphi Y, P Z\right)+g\left(\tilde{\nabla}_{X} \varphi Y, F Z\right) \\
& =-g\left(\tilde{\nabla}_{X} P Z, \varphi Y\right)+g(h(X, \varphi Y), F Z) \\
& =g\left(\varphi \tilde{\nabla}_{X} P Z, Y\right)+g(h(X, \varphi Y), F Z) .
\end{aligned}
$$$$
g\left(\nabla_{X} X Z\right)=g\left(\tilde{\nabla}_{X} \varphi P Z, Y\right)-g\left(\left(\tilde{\nabla}_{X} \varphi\right) P Z, Y\right)+g(h(X, \varphi Y), F Z) .
$$

Using (2.3), (2.8) and the orthogonality of vector fields, we obtain

$$
g\left(\nabla_{X} Y, Z\right)=g\left(\tilde{\nabla}_{X} P^{2} Z, Y\right)+g\left(\tilde{\nabla}_{X} F P Z, Y\right)+g(h(X, \varphi Y), F Z) .
$$

Then, from (2.11) and (2.6), we have

$$
\begin{aligned}
g\left(\nabla_{X} Y, Z\right)= & -\cos ^{2} \theta g\left(\tilde{\nabla}_{X} Z, Y\right)+\sin 2 \theta X(\theta) g(Y, Z)-g(h(X, Y), F P Z) \\
& +g(h(X, \varphi Y), F Z) .
\end{aligned}
$$

From the orthogonality of the two distributions the above equation takes the form

$$
g\left(\nabla_{X} Y, Z\right)=\cos ^{2} \theta g\left(\tilde{\nabla}_{X} Y, Z\right)-g(h(X, Y), F P Z)+g(h(X, \varphi Y), F Z) .
$$


Hence, (i) follows from the above relation. In a similar way we can prove (ii).

\section{Warped Product Pointwise Semi-Slant Submanifolds}

In this section, we study warped product submanifolds of Sasakian manifolds, by considering that one factor is a pointwise slant submanifold. In the following, first we give a brief introduction on warped product manifolds.

In [3], R. L. Bishop and B. O'Neill introduced the notion of warped product manifolds as follows: Let $M_{1}$ and $M_{2}$ be two Riemannian manifolds with Riemannian metrics $g_{1}$ and $g_{2}$, respectively, and a positive differentiable function $f \circ M_{1}$. Consider the product manifold $M_{1} \times M_{2}$ with its projections $\pi_{1}: M_{1} \times M_{2} \rightarrow M_{1}$ and $\pi_{2}: M_{1} \times M_{2} \rightarrow M_{2}$. Then their warped product manifold $M=M_{1} \times M_{2}$ is the Riemannian manifold $M_{1} \times M_{2}=\left(M_{1} \times M_{2}, g\right)$ equipped with the Riemannian metric

$$
g(X, Y)=g_{1}\left(\pi_{1 \star} X, \pi_{1 \star} Y\right)+\left(f \circ \pi_{1}\right)^{2} g_{2}\left(\pi_{2 \star} X, \pi_{2 \star} Y\right)
$$

for any vector field $X, Y$ tangent to $M$, where $\star$ is the symbol for the tangent maps. A warped product manifold $M=M_{1} \times_{f} M_{2}$ is said to betrivial or simply a Riemannian product manifold if the warping function $f$ is constant.

Let $X$ be a vector field tangent to $M_{1}$ and $Z$ be an another vector field on $M_{2}$; then from Lemma 7.3 of [3], we have

$$
\nabla_{X} Z=\nabla_{Z}
$$

where $\nabla$ is the Levi-Civita connection on $M$. If $M=M_{1} \times_{f} M_{2}$ is a warped product manifold then the base manifol $M_{1}$ is totally geodesic in $M$ and the fiber $M_{2}$ is totally umbilical in $M[3,9]$

By analogy to CR-warped products which are introduced by B.-Y. Chen in [9], one defines the warped product pointwise semi-slant submanifolds as follows.

Definition 4.1. A warped product of an invariant and a pointwise slant submanifolds, say $M_{T}$ and $M_{\theta}$ of a Sasakian manifold $\tilde{M}$ is called a warped product pointwise semislant submanifold.

A warped product pointwise semi-slant submanifold is called proper if $M_{\theta}$ is a proper pointwise slant submanifold and $M_{T}$ is an invariant submanifold of $\tilde{M}$.

The non-existence of warped product pointwise semi-slant submanifolds of the form $M_{\theta} \times_{f} M_{T}$ in Kaehler manifolds is proved in [26]. A similar result holds in Sasakian manifolds. On the other hand, there exist non-trivial warped product pointwise semislant submanifolds of the form $M_{T} \times M_{\theta}$ of Kaehler manifolds [26] and contact metric manifolds.

Note that a warped product pointwise semi-slant submanifold $M=M_{T} \times_{f} M_{\theta}$ is a warped product contact CR-submanifold if the slant function $\theta=\frac{\pi}{2}$. Similarly, the warped product pointwise semi-slant submanifold $M=M_{T} \times_{f} M_{\theta}$ is a warped product semi-slant submanifold if $\theta$ is constant on $M$, i.e., $M_{\theta}$ is a proper slant submanifold. 
In this section, we study the warped product pointwise semi-slant submanifold of the form $M=M_{T} \times_{f} M_{\theta}$ of a Sasakian manifold $\tilde{M}$. To fill the gap in the earlier study, we obtain some results as a generalization.

On a warped product pointwise semi-slant submanifold $M=M_{T} \times_{f} M_{\theta}$, if we consider the structure vector field $\xi$ tangent to $M$, then either $\xi \in \Gamma\left(T M_{T}\right)$ or $\xi \in \Gamma\left(T M_{\theta}\right)$. When $\xi$ is tangent to $M_{\theta}$, then it is easy to check that warped product is trivial (see [27]); therefore we always consider $\xi \in \Gamma\left(T M_{T}\right)$.

First, we prove the following useful results.

Lemma 4.1. Let $M=M_{T} \times_{f} M_{\theta}$ be a warped product pointwise semi-slant submanifold of a Sasakian manifold $\tilde{M}$ such that $\xi \in \Gamma\left(T M_{T}\right)$, where $M_{T}$ is an invariant submanifold and $M_{\theta}$ is a proper pointwise slant submanifold of $\tilde{M}$. Then, we have

$$
g(h(X, W), F P Z)-g(h(X, P Z), F W)=\sin 2 \theta X(\theta) g(Z, h
$$

for any $X \in \Gamma\left(T M_{T}\right)$ and $Z, W \in \Gamma\left(T M_{\theta}\right)$.

Proof. For any $X \in \Gamma\left(T M_{T}\right)$ and $Z, W \in \Gamma\left(T M_{\theta}\right)$,

$$
g\left(\tilde{\nabla}_{X} Z, W\right)=X(\ln f)
$$

On the other hand, we can obtain $g\left(\tilde{\nabla}_{X} Z, W\right)=g\left(\varphi \tilde{\nabla}_{X} Z, \varphi W\right)$. Using the covariant derivative formula of $\varphi$, we get

$$
g\left(\tilde{\nabla}_{X} Z, W\right)=g\left(\tilde{\nabla}_{X} \varphi Z \varphi W\right)-g\left(\left(\tilde{\nabla}_{X} \varphi\right) Z, \varphi W\right) .
$$

The second term in the right hand side of above relation is identically zero by using (2.3) and the orthogonality of yector fields. Then, from (2.5), (2.8), (4.1) and the orthogonality of vector fields, we fine

$$
\begin{aligned}
g\left(\tilde{\nabla}_{X} Z, W\right)= & g\left(\tilde{\nabla}_{X} P Z P W\right)+g\left(\tilde{\nabla}_{X} P Z, F W\right)+g\left(\tilde{\nabla}_{X} F Z, \varphi W\right) \\
= & X(\ln f) g(P Z, P W)+g(h(X, P Z), F W)-g\left(\varphi \tilde{\nabla}_{X} F Z, W\right) \\
= & \cos ^{2} \theta X(\ln f) g(Z, W)+g(h(X, P Z), F W)-g\left(\tilde{\nabla}_{X} \varphi F Z, W\right) \\
& +g\left(\left(\tilde{\nabla}_{X} \varphi\right) F Z, W\right) .
\end{aligned}
$$

Again, the last term in the above equation is zero by using (2.3) and the orthogonality of vector fields. Then, from (2.9) and (2.14), we derive

$$
\begin{aligned}
g\left(\tilde{\nabla}_{X} Z, W\right)= & \cos ^{2} \theta X(\ln f) g(Z, W)+g(h(X, P Z), F W)+\sin ^{2} \theta g\left(\tilde{\nabla}_{X} Z, W\right) \\
& +\sin 2 \theta X(\theta) g(Z, W)+g\left(\tilde{\nabla}_{Z} F P X, Y\right) .
\end{aligned}
$$

Hence, the result follows from (4.3) and (4.4) by using (2.6)-(2.7) and (4.1).

Lemma 4.2. Let $M=M_{T} \times_{f} M_{\theta}$ be a warped product pointwise semi-slant submanifold of a Sasakian manifold $\tilde{M}$ such that $\xi \in \Gamma\left(T M_{T}\right)$, where $M_{T}$ and $M_{\theta}$ are invariant and pointwise slant submanifolds of $\tilde{M}$, respectively. Then

(i) $g(P Z, W)=-\xi(\ln f) g(Z, W)$;

(ii) $g(h(X, Y), F Z)=0$; 
(iii) $g(h(X, Z), F W)=X(\ln f) g(P Z, W)-\varphi X(\ln f) g(Z, W)-\eta(X) g(Z, W)$, for any $X, Y \in \Gamma\left(T M_{T}\right)$ and $Z, W \in \Gamma\left(T M_{\theta}\right)$.

Proof. From (2.4), (2.5) and (2.8), we have $\nabla_{Z} \xi=-P Z$, for any $Z \in \Gamma\left(T M_{\theta}\right)$. Using (4.1) and taking the inner product with $W \in \Gamma\left(T M_{\theta}\right)$, we get (i). For the other parts of the lemma, considering any $X, Y \in \Gamma\left(T M_{T}\right)$ and $Z \in \Gamma\left(T M_{\theta}\right)$, we have

$$
g(h(X, Y), F Z)=g\left(\tilde{\nabla}_{X} Y, F Z\right)=g\left(\tilde{\nabla}_{X} Y, \varphi Z\right)-g\left(\tilde{\nabla}_{X} Y, P Z\right) .
$$

From (2.2) and (4.1), we get

$$
g(h(X, Y), F Z)=-g\left(\varphi \tilde{\nabla}_{X} Y, Z\right)+X(\ln f) g(Y, P Z)
$$

By covariant derivative formula of $\varphi$ and the orthogonality of vector fields, we find

$$
g(h(X, Y), F Z)=g\left(\left(\tilde{\nabla}_{X} \varphi\right) Y, Z\right)-g\left(\tilde{\nabla}_{X} \varphi Y Z\right)
$$

Using (2.3) and the fact that $\xi \in \Gamma\left(T M_{T}\right)$, the first term the Ningt tand side of above equation vanishes identically and then by using (4.1) and the orthogonality of vector fields, we find (ii). Now, for any $X \in \Gamma\left(T M_{T}\right)$ and $Z, W \in \Gamma\left(T M_{\theta}\right)$, we have

$$
g(h(X, Z), F W)=g\left(\tilde{\nabla}_{Z} X, F W\right)=g\left(\tilde{\nabla}_{X} Z, \varphi W\right)-g\left(\tilde{\nabla}_{X} Z, P W\right) .
$$

Again, using the covariant derivative formula of the Rientannain connection and (4.1), we get

$$
g(h(X, Z), F W)=g\left(\left(\tilde{\nabla}_{Z} \varphi\right) X, W\right)-g\left(\tilde{\nabla}_{z} \varphi X, W\right)-X(\ln f) g(Z, P W) .
$$

Then from (2.3), (2.5) and (4.1), we derive

$$
g(h(X, Z), F W)=-\eta(X) g(Z, W-\varphi X(\ln f) g(Z, W)-X(\ln f) g(Z, P W),
$$

which is the third part of the lemma. Hence, the proof is complete.

Lemma 4.3. Let $M=M_{T} \times_{f} M_{\theta}$ be a warped product pointwise semi-slant submanifold of a Sasakian manifold $\widetilde{M}$ such that $\xi \in \Gamma\left(T M_{T}\right)$, where $M_{T}$ is an invariant submanifold and $\mathbb{M}_{\theta}$ is a paintwise slant submanifold of $\tilde{M}$. Then for any $X \in \Gamma\left(T M_{T}\right)$ and $Z, W \in \Gamma\left(T M_{\theta}\right)$.

Proof. Interchanging $X$ by $\varphi X$, for any $X \in \Gamma\left(T M_{T}\right)$ in Lemma 4.2 (iii) and using the first part of Lemma 4.2, we get the required result.

Lemma 4.4. Let $M=M_{T} \times_{f} M_{\theta}$ be a warped product pointwise semi-slant submanifold of a Sasakian manifold $\tilde{M}$ such that $\xi \in \Gamma\left(T M_{T}\right)$, where $M_{T}$ and $M_{\theta}$ are invariant and pointwise slant submanifolds of $\tilde{M}$, respectively. Then, we have

$$
g(h(X, P Z), F W)=\varphi X(\ln f) g(Z, P W)-\eta(X) g(P Z, W)-\cos ^{2} \theta X(\ln f) g(Z, W),
$$

for any $X \in \Gamma\left(T M_{T}\right)$ and $Z, W \in \Gamma\left(T M_{\theta}\right)$. 
Proof. Interchange $Z$ by $P Z$, for any $Z \in \Gamma\left(T M_{\theta}\right)$ in Lemma 4.2 (iii) and after using (2.12), we get (4.6).

Similarly, if we interchange $W$ by $P W$, for any $W \in \Gamma\left(T M_{\theta}\right)$ in Lemma 4.2 (iii), then we can obtain the following result.

Lemma 4.5. Let $M=M_{T} \times_{f} M_{\theta}$ be a warped product pointwise semi-slant submanifold of a Sasakian manifold $\tilde{M}$ such that $\xi \in \Gamma\left(T M_{T}\right)$, where $M_{T}$ and $M_{\theta}$ are invariant and pointwise slant submanifolds of $\tilde{M}$, respectively. Then

$$
g(h(X, Z), F P W)=\cos ^{2} \theta X(\ln f) g(Z, W)-\varphi X(\ln f) g(Z, P W)-n(X) g(Z, P W),
$$

for any $X \in \Gamma\left(T M_{T}\right)$ and $Z, W \in \Gamma\left(T M_{\theta}\right)$.

Lemma 4.6. Let $M=M_{T} \times{ }_{f} M_{\theta}$ be a warped product pointwise semislant submanifold of a Sasakian manifold $\tilde{M}$ such that $\xi \in \Gamma\left(T M_{T}\right)$, where $M_{P}$ and $M_{\theta}$ are invariant and proper pointwise slant submanifolds of $\tilde{M}$, respectively. Then, we have

$$
g\left(A_{F W} \varphi X, Z\right)-g\left(A_{F P W} X, Z\right)=\sin ^{2} \theta X(\ln f) g(Z, W),
$$

for any $X \in \Gamma\left(T M_{T}\right)$ and $Z, W \in \Gamma\left(T M_{\theta}\right)$.

Proof. Subtracting (4.7) from (4.5), we

A warped product submanifold $M=M_{1} \gamma_{f}$ of a Sasakian manifold $\tilde{M}$ is said to be mixed totally geodesic if $h(X, Z)=0$, for any $X \in \Gamma\left(T M_{1}\right)$ and $Z \in \Gamma\left(T M_{2}\right)$, where $M_{1}$ and $M_{2}$ are any Riemannian submanifolds of $\tilde{M}$.

From Lemma 4.6, we obtain the following result.

Theorem 4.1. Let $M=M_{T} \times{ }_{f} M_{\theta}$ be a warped product pointwise semi-slant submanifold of a Sasakian manifold $\tilde{M}$. If $M$ is mixed totally geodesic, then either $M$ is warped product of invariant subranifolds or the warping function $f$ is constant on $M$.

Proof. From (4.8) and the mixed totally geodesic condition, we have

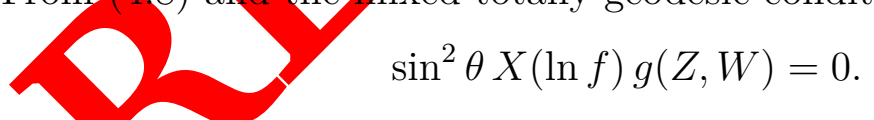

Since $g$ is a Rlemannian metric, then either $\sin ^{2} \theta=0$ or $X(\ln f)=0$. Therefore, either $M$ is warped product of invariant submanifolds or $f$ is constant on $M$, thus, the proof is complete.

Lemma 4.7. Let $M=M_{T} \times_{f} M_{\theta}$ be a warped product pointwise semi-slant submanifold of a Sasakian manifold $\tilde{M}$ such that $\xi \in \Gamma\left(T M_{T}\right)$, where $M_{T}$ and $M_{\theta}$ are invariant and pointwise slant submanifolds of $\tilde{M}$, respectively. Then, we have

$$
g\left(A_{F P Z} W, X\right)-g\left(A_{F W} P Z, X\right)=2 \cos ^{2} \theta X(\ln f) g(Z, W),
$$

for any $X \in \Gamma\left(T M_{T}\right)$ and $Z, W \in \Gamma\left(T M_{\theta}\right)$. 
Proof. Interchanging $Z$ and $W$ in (4.7) and using (2.10), we get $g(h(X, W), F P Z)=\cos ^{2} \theta X(\ln f) g(Z, W)+\varphi X(\ln f) g(Z, P W)+\eta(X) g(Z, P W)$, for any $X \in \Gamma\left(T M_{T}\right)$ and $Z, W \in \Gamma\left(T M_{\theta}\right)$. Subtracting (4.6) from (4.10), we find (4.9).

Also, with the help of Lemma 4.7, we find the following result.

Theorem 4.2. Let $M=M_{T} \times_{f} M_{\theta}$ be a warped product pointwise semi-slant submanifold of a Sasakian manifold $\tilde{M}$. If $M$ is mixed totally geodesic, then either $M$ is a contact $C R$-warped product of the form $M_{T} \times_{f} M_{\perp}$ or the warping function $f$ is constant on $M$.

Proof. From (4.9) and the mixed totally geodesic condition, wate

$$
\cos ^{2} \theta X(\ln f) g(Z, W)=0
$$

Since $g$ is a Riemannian metric, then either $\cos ^{2} \theta=0$ or $X(\ln f)=0$. Therefore, either $M$ is a contact CR-warped product or $f$ is constant on $M$, which ends the proof.

From Theorem 4.1 and Theorem 4.2, we condude the following result.

Corollary 4.1. There does not exist any mixed totally geodesic proper warped product pointwise semi-slant submanifold $M=M_{T} x_{f} M_{\theta}$ of a Sasakian manifold.

Also, from Lemma 4.1 and Lemma 4.7, we have the following result.

Theorem 4.3. Let $M=M_{T} \times M_{f}$ be a warped product pointwise semi-slant submanifold of a Sasakian manifold $\tilde{M}$ such that $\xi \in \Gamma\left(T M_{T}\right)$, where $M_{T}$ is an invariant submanifold and $M_{\theta}$ a pointwise slant submanifold of $\tilde{M}$. Then, either $M$ is a contact CR-warped product of the form $M=M_{T} \times_{f} M_{\perp}$ or $\nabla(\ln f)=\tan \theta \nabla \theta$, for any $X \in \Gamma\left(T M_{T}\right)$, where $\nabla f$ is the gradient of $f$.

Proof. From Lemma 4 1 and Lemma 4.7, we have

$$
\cos ^{2} \theta\{X(\ln f)-\tan \theta X(\theta)\} g(Z, W)=0 .
$$

Since $g$ is a Riemannian metric, therefore, we conclude that either $\cos ^{2} \theta=0$ or $X(\ln f)-\tan \theta X(\theta)=0$. Consequently, either $\theta=\frac{\pi}{2}$ or $X(\ln f)=\tan \theta X(\theta)$, which proves the theorem completely.

As an application of Theorem 4.3, we have the following consequence.

Remark 4.1. If we consider that the slant function $\theta$ is constant, i.e., $M_{\theta}$ is a proper slant submanifold in Theorem 4.3, then $Z(\ln f)=0$, i.e., there are no warped product semi-slant submanifolds of the form $M_{T} \times_{f} M_{\theta}$ in Sasakian manifolds. Hence, Theorem 3.3 of [1] is a special case of Theorem 4.3 . 
In order to give a characterization result for pointwise semi-slant submanifolds of a Sasakian manifold, we need the following well-known result of Hiepko [18].

Theorem 4.4 (Hiepko's Theorem). Let $\mathfrak{D}_{1}$ and $\mathfrak{D}_{2}$ be two orthogonal distribution on a Riemannian manifold $M$. Suppose that both $\mathfrak{D}_{1}$ and $\mathfrak{D}_{2}$ are involutive such that $\mathfrak{D}_{1}$ is a totally geodesic foliation and $\mathfrak{D}_{2}$ is a spherical foliation. Then $M$ is locally isometric to a non-trivial warped product $M_{1} \times{ }_{f} M_{2}$, where $M_{1}$ and $M_{2}$ are integral manifolds of $\mathfrak{D}_{1}$ and $\mathfrak{D}_{2}$, respectively.

Theorem 4.5. Let $M$ be a pointwise semi-slant submanifold of a Sasakian manifold $\tilde{M}$. Then $M$ is locally a non-trivial warped product submanifold of the form $M_{T} \times{ }_{f} M_{\theta}$, where $M_{T}$ is an invariant submanifold and $M_{\theta}$ is a proper pointwise slant submanifold of $\tilde{M}$ if and only if

$$
A_{F W} \varphi X-A_{F P W} X=\sin ^{2} \theta X(\mu) W, \quad \text { for all } X \in \Gamma(\mathfrak{D} \bullet
$$
for some smooth function $\mu$ on $M$ satisfying $Z(\mu)=0$ for any $Z \in \Gamma\left(D^{\circ}\right)$.

Proof. Let $M=M_{T} \times{ }_{f} M_{\theta}$ be a warped product pointwise semi-slant submanifold of a Sasakian manifold $\tilde{M}$. Then for any $X \in \Gamma\left(T M_{T}\right)$ and $Z, W \in \Gamma\left(T M_{\theta}\right)$, from Lemma 4.2 (ii) we have

$$
g\left(A_{F W} X, Y\right)
$$

Interchanging $X$ by $\varphi X$ in (4.12), we get $g\left(A_{F w} X, Y\right)=0$, which means that $A_{F W} \varphi X$ has no component in $T M_{\mathrm{T}}$. Simularly if we interchange $W$ by $P W$ in (4.12) then, we get $g\left(A_{F P W} X, Y\right)=0$, i.e., $A_{F P W} X$ also has no component in $T M_{T}$. Therefore, $A_{F W} \varphi X-A_{F P W}$ X lies in TM, using this fact with Lemma 4.6, we find (4.11).

Conversely, if $M$ is a pointwise semi-slant submanifold such that (4.11) holds, then from Lemma 3.1 (i) we have

$$
g\left(\nabla_{X} Y, W\right)=\csc ^{2} \theta g\left(A_{F W} \varphi Y-A_{F P W} Y, X\right),
$$

for any $X, Y \in \Gamma(\mathfrak{D} \bigcirc\langle\xi\rangle)$ and $W \in \Gamma\left(\mathfrak{D}^{\theta}\right)$. From (4.11), we arrive at

$$
\text { . } g\left(\nabla_{X} Y, W\right)=Y(\mu) g(X, W)=0,
$$

which means that the leaves of the distribution $\mathfrak{D} \oplus\langle\xi\rangle$ are totally geodesic in $M$. Also, from Lemma 3.1 (ii), we have

$$
g\left(\nabla_{Z} W, X\right)=\csc ^{2} \theta g\left(A_{F P W} X-A_{F W} \varphi X, Z\right),
$$

for any $Z, W \in \Gamma\left(\mathfrak{D}^{\theta}\right)$ and $X \in \Gamma(\mathfrak{D} \oplus\langle\xi\rangle)$. By polarization, we derive

$$
g\left(\nabla_{W} Z, X\right)=\csc ^{2} \theta g\left(A_{F P Z} X-A_{F Z} \varphi X, W\right) .
$$

Substracting (4.14) from (4.13), we get

$$
\sin ^{2} \theta g([Z, W], X)=g\left(A_{F Z} \varphi X-A_{F P Z} X, W\right)-g\left(A_{F W} \varphi X-A_{F P W} X, Z\right) .
$$


Using (4.11), we get

$$
\sin ^{2} \theta g([Z, W], X)=X(\mu) g(Z, W)-X(\mu) g(W, Z)=0 .
$$

Since $M$ is proper pointwise semi-slant, then $\sin ^{2} \theta \neq 0$, thus we conclude that the pointwise slant distribution $\mathfrak{D}^{\theta}$ is integrable. Let us consider $M_{\theta}$ to be a leaf of $\mathfrak{D}^{\theta}$ and $h^{\theta}$ is the second fundamental form of $M_{\theta}$ in $M$. Then from (4.14), we have

$$
g\left(h^{\theta}(Z, W), X\right)=g\left(\nabla_{Z} W, X\right)=-\csc ^{2} \theta g\left(A_{F W} \varphi X-A_{F P W} X, Z\right) .
$$

Using (4.11), we find that

$$
g\left(h^{\theta}(Z, W), X\right)=-X(\mu) g(Z, W) .
$$

Then from the definition of the gradient of a function, we arrive

$$
h^{\theta}(Z, W)=-(\vec{\nabla} \mu) g(Z, W) .
$$

Hence, $M_{\theta}$ is a totally umbilical submanifold of $M$ with the mean curyature vector $H^{\theta}=-\vec{\nabla} \mu$, where $\vec{\nabla} \mu$ is the gradient of the function $\mu$. Since $Z(\mu)=0$, for any $Z \in \Gamma\left(\mathfrak{D}^{\theta}\right)$, then we can show that $H^{\theta}=-\vec{\nabla} \mu$ is parallel with respect to the normal connection, say $D^{n}$ of $M_{\theta}$ in $M$ (see [25,26], [28). Thus, $M_{\theta}$ is a totally umbilical submanifold of $M$ with a non vanishing parallel mean curvature vector $H^{\theta}=-\vec{\nabla} \mu$, i.e., $M_{\theta}$ is an extrinsic sphere in $M$. Then from Heipko's Theorem [18], we conclude that $M$ is a warped product manifold of $M_{T}$ and $M_{\theta}$, where $M_{T}$ and $M_{\theta}$ are integral manifolds of $\mathfrak{D} \oplus\langle\xi\rangle$ and $\mathfrak{D}^{\theta}$, respectively. Thus, the proof is complete.

As an application of Theorem 4.5, if we consider $\theta=\frac{\pi}{2}$ in Theorem 4.5, then by interchanging $X$ by $\varphi X$ in (4,11) we get the condition (74) of Theorem 3.2 in [21]; thus the Theorem 4.5 is true for contact CR-warped product submanifolds of the form $M_{T} \times{ }_{f} M_{\perp}$. Hence, Theorem 3.2 of 21 jis a special case of Theorem 4.5 as follows.

Corollary 4.2 (Theorem 3.2 of [21]). A strictly proper CR-submanifold $M$ of a Sasakian manifold $\hat{M}$ tangent to the structure vector field $\xi$ is locally a contact $C R$ warped product if and onty if

for some function $\mu$ on $M$ satisfying $W \mu=0$, for all $W \in \Gamma\left(\mathfrak{D}^{\perp}\right)$.

\section{EXAMPLES}

In this section, we provide the following non-trivial examples of Riemannian products and warped product pointwise semi-slant submanifolds in Euclidean spaces.

Example 5.1. Let $M$ be a submanifold of Euclidean 7 -space $\mathbb{R}^{7}$ with its cartesian coordinates $\left(x_{1}, \ldots, x_{3}, y_{1}, \ldots, y_{3}, t\right)$ and the almost contact structure

$$
\varphi\left(\frac{\partial}{\partial x_{i}}\right)=-\frac{\partial}{\partial y_{i}}, \quad \varphi\left(\frac{\partial}{\partial y_{j}}\right)=\frac{\partial}{\partial x_{j}}, \quad \varphi\left(\frac{\partial}{\partial t}\right)=0, \quad 1 \leq i, j \leq 3 .
$$


If $M$ is given by the equations

$$
\begin{aligned}
& x_{1}=u_{1}, \quad x_{2}=u_{3} \cos u_{4}, \quad x_{3}=\frac{u_{3}^{2}}{2}, \quad y_{1}=u_{2}, \quad y_{2}=u_{3} \sin u_{4}, \\
& y_{3}=u_{4}, \quad t=t,
\end{aligned}
$$

for any non-zero function $u_{3}$ on $M$, then tangent space $T M$ of $M$ is spanned by $X_{1}, X_{2}, X_{3}, X_{4}$ and $X_{5}$, where

$$
\begin{aligned}
& X_{1}=\frac{\partial}{\partial x_{1}}, \quad X_{2}=\frac{\partial}{\partial y_{1}}, \quad X_{3}=\cos u_{4} \frac{\partial}{\partial x_{2}}+u_{3} \frac{\partial}{\partial x_{3}}+\sin u_{4} \frac{\partial}{\partial y_{2}}, \\
& X_{4}=-u_{3} \sin u_{4} \frac{\partial}{\partial x_{2}}+u_{3} \sin u_{4} \frac{\partial}{\partial y_{2}}+\frac{\partial}{\partial y_{3}}, \quad X_{5}=\frac{\partial}{\partial t} .
\end{aligned}
$$

Then, $M$ is a pointwise semi-slant submanifold with invariant distribution $\mathfrak{D}=$ $\operatorname{Span}\left\{\mathrm{X}_{1}, \mathrm{X}_{2}\right\}$ and the pointwise slant distribution $\mathfrak{D}^{\theta}=\operatorname{Span}\left\{X_{3}, X_{4}\right\}$ Clearly, the slant function is $\theta=\cos ^{-1}\left(2 u_{3} / \sqrt{1+u_{3}^{2}}\right)$. Moreover, $\mathfrak{D}$ and $\mathfrak{D}^{\theta}$ are integrable. If $M_{T}$ and $M_{\theta}$ are integral manifolds of $\mathfrak{D}$ and $\mathfrak{D}^{\theta}$, respectirely, then, $M=M_{T} \times M_{\theta}$ is a Riemannian product of $M_{T}$ and $M_{\theta}$ in $\mathbb{R}^{9}$.

Example 5.2. Consider the Euclidean 9-space $\mathbb{R}^{9}$ with its Cartesian coordinates $\left(x_{1}, \ldots, x_{4}, y_{1}, \ldots, y_{4}, t\right)$ and the almost contact structure

$$
\varphi\left(\frac{\partial}{\partial x_{i}}\right)=-\frac{\partial}{\partial y_{i}}, \quad \varphi\left(\frac{\partial}{\partial y_{j}}\right)=\frac{\partial}{\partial x_{2}} \varphi\left(\frac{\partial}{\partial t}\right)=0, \quad 1 \leq i, j \leq 4 .
$$

Let $M$ be a submanifold of $\mathbb{R}^{9}$ defined by the immersion $\psi$ as follows:

$$
\psi(u, v, w, s, t)=\left(u+v, \frac{1}{2} w^{2}, s \cos w \sin w,-u+v, \frac{1}{2} s^{2},-w \sin s, w \cos s, t\right),
$$

for any non-zero real numbers and $s$. The tangent space of $M$ is spanned by the following vectors

$$
\begin{aligned}
& X_{1}=\frac{\partial}{\partial x_{1}} \frac{\partial}{\partial y_{1}} X_{2}=\frac{\partial}{\partial x_{1}}+\frac{\partial}{\partial y_{1}}, \\
& X_{3}=w \frac{\partial}{\partial x_{2}} w \frac{\partial}{\partial x_{3}}+s \cos w \frac{\partial}{\partial x_{4}}-\sin s \frac{\partial}{\partial y_{3}}+\cos v \frac{\partial}{\partial y_{4}}, \\
& X_{4}=\cos w \frac{\partial}{\partial x_{3}}+\sin w \frac{\partial}{\partial x_{4}}+s \frac{\partial}{\partial y_{2}}-w \cos s \frac{\partial}{\partial y_{3}}-w \sin s \frac{\partial}{\partial y_{4}}, \quad X_{5}=\frac{\partial}{\partial t} .
\end{aligned}
$$

Then, $M$ is a pointwise semi-slant submanifold such that the structure vector field $\xi=\frac{\partial}{\partial t}$ is tangent to $M$ and $\mathfrak{D}=\operatorname{Span}\left\{X_{1}, X_{2}\right\}$ is an invariant distribution and $\mathfrak{D}^{\theta}=$ $\operatorname{Span}\left\{X_{3}, X_{4}\right\}$ is a pointwise slant distribution with slant function $\theta=\cos ^{-1}\left(\frac{(1-w s) \sin (w-s)-w s}{1+w^{2}+s^{2}}\right)$. It is easy to observe that both the distributions are integrable. If we denote the integral manifolds of $\mathfrak{D}$ and $\mathfrak{D}^{\theta}$ by $M_{T}$ and $M_{\theta}$, respectively, then $M$ is a Riemannian product of invariant and pointwise slant submanifolds in $\mathbb{R}^{9}$. 
Example 5.3. Let $M$ be a submanifold of $\mathbb{R}^{13}$ given by the immersion $\psi: \mathbb{R}^{5} \rightarrow \mathbb{R}^{13}$ as follows:

$$
\begin{aligned}
\psi\left(u_{1}, v_{1}, u_{2}, v_{2}, t\right)= & \left(u_{1}-v_{1}, u_{1} \cos \left(u_{2}+v_{2}\right), u_{1} \sin \left(u_{2}+v_{2}\right), v_{2}, u_{1} \cos \left(u_{2}-v_{2}\right),\right. \\
& u_{1} \sin \left(u_{2}-v_{2}\right), u_{1}+v_{1}, v_{1} \cos \left(u_{2}+v_{2}\right), v_{1} \sin \left(u_{2}+v_{2}\right), u_{2}, \\
& \left.v_{1} \cos \left(u_{2}-v_{2}\right), v_{1} \sin \left(u_{2}-v_{2}\right), t\right),
\end{aligned}
$$

for non-zero functions $u_{1}$ and $v_{1}$. We use the almost contact structure from Example 5.2. Then, we have

$$
\begin{aligned}
X_{1}= & \frac{\partial}{\partial x_{1}}+\cos \left(u_{2}+v_{2}\right) \frac{\partial}{\partial x_{2}}+\sin \left(u_{2}+v_{2}\right) \frac{\partial}{\partial x_{3}}+\cos \left(u_{2}-v_{2}\right) \frac{\partial}{\partial x_{5}} \\
& +\sin \left(u_{2}-v_{2}\right) \frac{\partial}{\partial x_{6}}+\frac{\partial}{\partial y_{1}}, \\
X_{2}= & -\frac{\partial}{\partial x_{1}}+\frac{\partial}{\partial y_{1}}+\cos \left(u_{2}+v_{2}\right) \frac{\partial}{\partial y_{2}}+\sin \left(u_{2}+v_{2}\right) \frac{\partial}{\partial y_{3}}+\cos \left(u_{2}-v_{2}\right) \frac{\partial}{\partial y_{5}} \\
& +\sin \left(u_{2}-v_{2}\right) \frac{\partial}{\partial y_{6}}, \\
X_{3}= & -u_{1} \sin \left(u_{2}+v_{2}\right) \frac{\partial}{\partial x_{2}}+u_{1} \cos \left(u_{2}+v_{2}\right) \frac{\partial}{\partial x_{3}}-u_{1} \sin \left(u_{2}-v_{2}\right) \frac{\partial}{\partial x_{5}} \\
& +u_{1} \cos \left(u_{2}-v_{2}\right) \frac{\partial}{\partial x_{6}}-v_{1} \sin \left(u_{2}+v_{2}\right) \frac{\partial}{\partial y_{2}},+v_{1} \cos \left(u_{2}+v_{2}\right) \frac{\partial}{\partial y_{3}} \\
& +\frac{\partial}{\partial y_{4}}-v_{1} \sin \left(u_{2}-v_{2}\right) \frac{\partial}{\partial y_{5}}+v_{1} \cos \left(u_{2}-v_{2}\right) \frac{\partial}{\partial y_{6}}, \\
X_{4}= & -u_{1} \sin \left(u_{2}+v_{2}\right) \frac{\partial}{\partial x_{2}}+u_{1} \cos \left(u_{2}+v_{2}\right) \frac{\partial}{\partial x_{3}}+\frac{\partial}{\partial x_{4}}+u_{1} \sin \left(u_{2}-v_{2}\right) \frac{\partial}{\partial x_{5}} \\
& -u_{1} \cos \left(u_{2} \frac{\partial}{2} v_{2}\right) \frac{\partial}{\partial x_{6}}-v_{1} \sin \left(u_{2}+v_{2}\right) \frac{\partial}{\partial y_{2}},+v_{1} \cos \left(u_{2}+v_{2}\right) \frac{\partial}{\partial y_{3}} \\
& +v_{1} \sin \left(u_{2}-v_{2}\right) \frac{\partial}{\partial y_{5}}-v_{1} \cos \left(u_{2}-v_{2}\right) \frac{\partial}{\partial y_{6}}, \\
& \therefore
\end{aligned}
$$

By easy and direct computations we find that $\mathfrak{D}=\operatorname{Span}\left\{X_{1}, X_{2}\right\}$ is an invariant distribution and $\mathfrak{D}^{\theta}=\operatorname{Span}\left\{X_{3}, X_{4}\right\}$ is a pointwise slant distribution with slant function $\theta=\cos ^{-1}\left(\frac{1}{1+2 u_{1}^{2}+2 v_{1}^{2}}\right)$. Hence, $M$ is a pointwise semi-slant submanifold of $\mathbb{R}^{13}$. It is easy to observe that both the distributions are integrable. If we denote the integral manifolds of $\mathfrak{D}$ and $\mathfrak{D}^{\theta}$ by $M_{T}$ and $M_{\theta}$, respectively, then the product metric structure of $M$ is given by

$$
g=4\left(d u_{1}^{2}+d v_{1}^{2}\right)+\left(1+2 u_{1}^{2}+2 v_{1}^{2}\right)\left(d u_{2}^{2}+d v_{2}^{2}\right)=g_{M_{T}}+f^{2} g_{M_{\theta}} .
$$


Hence, $M=M_{T} \times_{f} M_{\theta}$ is a warped product submanifold in $\mathbb{R}^{13}$ with warping function $f=\sqrt{1+2 u_{1}^{2}+2 v_{1}^{2}}$.

\section{REFERENCES}

[1] F. R. Al-Solamy and V. A. Khan, Warped product semi-slant submanifolds of a Sasakian manifold, Serdica Math. J. 34 (2008), 597-606.

[2] F. R. Al-Solamy, V. A. Khan and S. Uddin, Geometry of warped product semi-slant submanifolds of nearly Kaehler manifolds, Results Math. 71 (2017), 783-799.

[3] R. L. Bishop and B. O'Neill, Manifolds of negative curvature, Trans. Amer. Math. Soc. 145 (1969), 1-49.

[4] D. E. Blair, Contact Manifolds in Riemannian Geometry, Lecture Notes in Mathematics 509, Springer-Verlag, New York, 1976.

[5] J. L. Cabrerizo, A. Carriazo, L. M. Fernandez and M. Fernandez, Semi-slant submanifolds of a Sasakian manifold, Geom. Dedicata 78 (1999), 183-199.

[6] J. L. Cabrerizo, A. Carriazo, L. M. Fernandez and M. Fernandez, Sant submanifolds in Sasakian manifolds, Glasg. Math. J. 42 (2000), 125-138.

[7] B.-Y. Chen, Slant immersions, Bull. Aust. Math. Soc. 41 (1990), 135-147.

[8] B.-Y. Chen, Geometry of Slant Submanifolds, Katholieke Universiteit, Leuren, 1990.

[9] B.-Y. Chen, Geometry of warped product CR-submanifolds in Kaehler manifolds, Monatsh. Math. 133 (2001), 177-195.

[10] B.-Y. Chen, Geometry of warped product CR-submanifolds in Kaehler manifolds II, Monatsh. Math. 134 (2001), 103-119.

[11] B.-Y. Chen, Pseudo-Riemannian Geometry, $\delta$-Invariants and Applications, World Scientific, Hackensack, New Jersey, 2011.

[12] B.-Y. Chen, Geometry of warped product submanifolds: a survey, J. Adv. Math. Stud. 6(2) (2013), 1-43.

[13] B.-Y. Chen, Differential Geometry of Warped Product Manifolds and Submanifolds, World Scientific, Hackensack, New J

[14] B.-Y. Chen and O. Garay, Pointwise stant submanifolds in almost Hermitian manifolds, Turkish J. Math. 36 (2012), 630

[15] B.-Y. Chen and S. Uddin, Warped product pointwise bi-slant submanifolds of Kaehler manifolds, Publ. Math. Debrecen 92(1) (2018), 1-16.

[16] F. Etayo, On quasi-slant submanifolds of an almost Hermitian manifold, Publ. Math. Debrecen 53 (1998), 217-223

[17] I. Hasegawa and I. Mihai, Contact CR-warped product submanifolds in Sasakian manifolds, Geom. Dedicata 102 (2003), 143-150.

[18] S. Hepko, Eine inner kennzeichungder verzerrten produkte, Math. Ann. 241 (1979), 209-215.

[19] K. A. Khan, V. A. Khan and S. Uddin, Warped product submanifolds of cosymplectic manifolds, Balkan J. Geom.Appl. 13 (2008), 55-65.

[20] A. Lotta, Slant submanifolds in contact geometry, Bull. Math. Soc. Roumanie 39 (1996), 183198.

[21] M. I. Munteanu, Warped product contact CR-submanifolds of Sasakian space forms, Publ. Math. Debrecen 66 (2005), 75-120.

[22] N. Papaghiuc, Semi-slant submanifolds of Kaehlerian manifold, Ann. Şt. Univ. Iaşi 9 (1994), 55-61.

[23] K. S. Park, Pointwise almost h-semi-slant submanifolds, Int. J. Math. 26 (2015), Article ID 1550099 .

[24] B. Sahin, Non existence of warped product semi-slant submanifolds of Kaehler manifolds, Geom. Dedicata 117 (2006), 195-202. 
[25] B. Sahin, Warped product submanifolds of Kaehler manifolds with a slant factor, Ann. Pol. Math. 95 (2009), 207-226.

[26] B. Sahin, Warped product pointwise semi-slant submanifolds of Kaehler manifolds, Port. Math. 70 (2013), 252-268.

[27] S. Uddin, V. A. Khan and H. H. Khan, Some results on warped product submanifolds of a Sasakian manifold, Int. J. Math. Math. Sci. (2010), Article ID 743074 DOI 10.1155/2010/743074.

[28] S. Uddin and F. R. Al-Solamy, Warped product pseudo-slant submanifolds of cosymplectic manifolds, An. Ştiinţ. Univ. Al. I. Cuza Iaşi Mat (N.S.) Tome LXIII, 3 (2016), 901-913.

[29] S. Uddin and F. R. Al-Solamy, Warped product pseudo-slant immersions in Sasakian manifolds, Publ. Math. Debrecen 91(3-4) (2017), 331-348.

[30] S. Uddin, B.-Y. Chen and F. R. Al-Solamy, Warped product bi-slant immersions in Kaehler manifolds, Mediterr. J. Math. 14(95) (2017), DOI 10.1007/s00009-017-0896-8

[31] S. Uddin and A. H. Alkhaldi, Pointwise slant submanifolds and their warped products in Sasakian manifolds, Filomat 32(12) (2018), 1-12.

[32] K. Yano and M. Kon, Structures on Manifolds, Series in Pure Mathematics, World Seientific Publishing Co., Singapore, 1984.

${ }^{1}$ Faculty of Mathematics, UNIVERSITY OF BUCHAREST, Str. ACAdemiei 14, 010014 Bucharest, Romania Email address: imihai@fmi.unibuc.ro

${ }^{2}$ Department of Mathematics, Faculty of Science, King AbDulaziz University, 21589 Jeddah, SAUdi Arabia Email address: siraj.ch@gmail.com

${ }^{3}$ Department of Mathematics and Compdter Sorence, TECHNiCAL UNIVERSITY OF CIVIL ENGINEERINGBUCHAREST, LACUl Tei Bvd. 122-124, 020396 Bucharest, Romania Email address: adela.mihai@utcb. 